\title{
The estimation of bubble-size spectra and its error analysis
}

\author{
TENG Yuehui ${ }^{1, a}$, HUANG Chun ${ }^{1, b}$ and FAN Wei $\mathrm{i}^{2, \mathrm{c}}$ \\ ${ }^{1}$ Science and Technology on Underwater Acoustic Antagonizing Laboratory, Shanghai, 201108, \\ China \\ ${ }^{2}$ Shanghai Marine Electronic Equipment Research Institute, Shanghai, 201108, China \\ atzihuy@163.com, bpure0304@sina.com, 'fanwei1029@126.com
}

Keywords: Bubble, Bubble-size spectra, Resonance, Estimated error

\begin{abstract}
The issues of bubble-size spectra estimation were researched using the bubble resonance theory and an iterative approximation, in order to further understanding the performance of the two approaches for determining bubble-size spectra, the estimation accuracy of four bubble-size spectra models have been analyzed. It comes out that the bubbles with medium range of bubble-size spectra can be precisely determined using the two approaches, however, the bubbles distributed at the two-end of bubble-size spectra have poor performance. The iterative approach ,by which the mean of bubble-size spectra estimation error less than 3\%,is significantly better for estimating bubble-size spectra than the bubble resonance, by which the mean of bubble-size spectra estimation error as much as $20 \%$, meanwhile the iterative approach with smaller variation of the estimation error. Therefore the iterative approach has a better accuracy for determining bubble-size spectra than the resonance.
\end{abstract}

\section{Introduction}

It is well known that the presence of bubbles in a liquid has a tremendous impact on its acoustical property. As documented [1,2,3], the bubbles can reach as much as 10 meters beneath sea surface and the void fraction of bubbles in water can reach as high as $10^{-3}$. The presence of bubbles will significantly change the properties of bubbly water, and profoundly impact the sound propagation in bubbly water because that its scattering section is much larger than its geometric section. Therefore, the acoustic properties of bubbles in bubbly water have attracted a lot of attention of researchers.

As early in 1933, the natural frequency of bubble was derived by M.Minnaert, which is expressed by $\omega_{\mathrm{M}}=\sqrt{3 \gamma \mathrm{P}_{\text {liq }} / \rho_{\text {liq }}} / \mathrm{R}_{0}$, later, more complicated factors including the frequency of driving pulse, the viscosity of water, the depth and size of bubbles, were taken into consideration in the literatures by Charles Devin[4], Marshall V. Hall[5] and Michael A. Ainsile[6], details of which will be depicted in subsequent sections.

Although the vibrations of resonant bubble have been rigorously deducted, the bubble population distributed in bubbly water can't be precisely determined, because the integration of all size bubbles is unpractical. Fortunately, the scattering section of bubble is much larger than its geometric section, based on this notion, the bubble population distributed in bubbly water approximated with barely taken the resonant bubbles into account was provided by Medwin[7], $\alpha(f)=8.686 \pi^{2} a_{R}^{3} n\left(a_{R}\right) / \delta_{R}$, which brings un-accuracy in some extent on the bubble population. Therefore, the improvement of the bubble population estimation with an iterative approach was presented by J. W. Caruthers and P. A. Elmore[8], which is also based on the bubble resonance.

Another impact of bubbles on sound wave propagation in bubbly water observed[1,9], is the phase speed of sound wave, which is a function of bubble population, and could be used for determining the bubble population using an inverse solution. Above all, it is assumed that the positions of bubbles in bubbly water are independent from each other, however, bubbles within naturally occurring clouds may be influenced by the dynamics of the fluids, in which they are entrained so that they become preferentially concentrated, or clustered, so a correlation function 
describing the relationship between the positions of the bubbles has been used for examining the errors associated with coherent structure in the fluctuating bubble density by Thomas C. Weber[10].

As an inverse problem, the bubble-size spectra have been modeled in a lot of literatures. In 1977, Medwin formed the bubble-size spectra as a power-law with bubble radii, the index between - 6 to -2[7], and later, more impact factors including the wind speed, the depth of bubbles, the bubble population and the variant with the depth were taken into the bubble-size spectra model, $N(a)=\mathrm{N}_{0} \mathrm{G}(\mathrm{a}, \mathrm{z}) \mathrm{U}(\mathrm{w}) \mathrm{Y}(\mathrm{z}, \mathrm{w})$, by Marshall V. Hall[5] and Novarini[11]. In order to investigate the influence of ship wakes on sound wave propagation, a ship wake model was constructed by B.R.Rapids[12] using the data measured by Mark V. Trevorrow[13].

Although the properties of bubbles within bubbly water have been well studied over the last century, the bubble-size spectra estimation is still a prospective topic in underwater acoustic, because of its wide applications. In this paper, we described the problem of counting bubbles within bubbly water and the reliability of the bubble-size spectra estimation. In Section I, the existing bubble-size spectra models were briefly introduced, and the theories for estimating the bubble-size spectra were introduced in Section II, then in Section III, the estimation errors of bubble-size spectra were analyzed, and the last is the summary of this paper.

\section{The bubble-size spectra models}

As mentioned in the introduction, several bubble-size spectra models have been presented, of which the four bubble-size spectra models were adopted as the object to analyze.

\section{Medwin-Breit model}

In 1982, Medwin and Breit summarized the near surface bubble-size spectra model using experimental data, which is suitable for the bubbles, whose radii is within $30 \mu \mathrm{m} \sim 270 \mu \mathrm{m}$, and can be written shortly as follows:

$$
N(a)=7.8 \times 10^{8}[a / 1 \mu m]^{-2.7} \quad 30 \mu m \leq a \leq 270 \mu m
$$

where $N(a)$ is the bubble-size spectra within bubbly water along radii, as shown in Fig.1, and $a$ is the radius of the bubble near sea surface, in micrometer.

\section{Marshall V. Hall model}

In 1989, based on the experimental data measured by Johnson, Cooke and Thorp, another bubble-size spectra model near surface was given.

$$
N(a)=\mathrm{N}_{0} \mathrm{G}(\mathrm{a}, \mathrm{z}) \mathrm{U}(\mathrm{w}) \mathrm{Y}(\mathrm{z}, \mathrm{w}) \quad 16 \mu \mathrm{m} \leq a \leq 1000 \mu \mathrm{m}
$$

Eq. 2 is suitable for the bubble of radii within $16 \mu \mathrm{m} \sim 1000 \mu \mathrm{m}, \mathrm{N}_{0}$ is the bubble population near surface, in the MarshallV. Hall model

$$
\mathrm{N}_{0}=1.6 \times 10^{10} \mathrm{~m}^{-4}
$$

$\mathrm{G}(\mathrm{a}, \mathrm{z})$, the distribution of bubbles is a function of bubble size and the depth of bubble, indicated by Eq. 4.

$$
\mathrm{G}(\mathrm{a}, \mathrm{z})=\left\{\begin{array}{cc}
\left(a / a_{1}\right)^{2} & a<a_{1} \\
1 & a_{1} \leq a \leq a_{2} \\
\left(a_{2} / a\right)^{p} & a>a_{2}
\end{array}\right.
$$

where $a_{1}=(34+1.24 \mathrm{z}) \times 10^{-6}(\mathrm{~m}), a_{2}=1.6 a_{1}(\mathrm{~m})$, and the index $p$ is a function of bubble depth in water, $p=4.37+(z / 2.55)^{2}$.

$$
\mathrm{U}(\mathrm{w})=(\mathrm{w} / 13)^{3}
$$

The influence of wind on bubbles distribution $U(w)$, increases with the cubic of its speed, $w$ in meter per second.

$$
\mathrm{Y}(\mathrm{z}, \mathrm{w})=\exp (-\mathrm{z} / \mathrm{L}(\mathrm{w}))
$$


The evolution of bubbles population $\mathrm{Y}(\mathrm{z}, \mathrm{w})$ in the vertical reduce with an exponential decay, where the exponential index

$$
L(w)=\left\{\begin{array}{lr}
0.4 & , w \leq 7.5 \mathrm{~m} / \mathrm{s} \\
0.4+0.115(w-7.5), w>7.5 \mathrm{~m} / \mathrm{s}
\end{array}\right.
$$

Thus, substitution of Eq.3, Eq.4, Eq.5, Eq.6 into Eq.2 will derive the bubble-size spectra model near surface. The Marshall V. Hall model was plotted in Fig.1.

\section{Novarini- $\beta$ and $\gamma$ model}

In 1998, Novarini presented a similar model with Marshall V. Hall model. The Novarini model can be divided into $\beta$-distribution and $\gamma$-distribution with the different bubble population. The Novarini- $\beta$ distribution is expressed as follows:

$$
\begin{aligned}
& \mathrm{N}_{\beta}=2.0 \times 10^{13} \mathrm{~m}^{-4} \\
& \mathrm{G}_{\beta}(\mathrm{a}, \mathrm{z})= \begin{cases}0, & a<a_{\text {min }} \\
\left(a / a_{1}\right)^{3}, & a_{\text {min }} \leq a<a_{1} \\
1, & a_{1} \leq a<a_{2} \\
\left(a / a_{2}\right)^{-4}, & a_{2} \leq a<a_{3}\end{cases} \\
& \mathrm{Y}_{\beta}(\mathrm{z}, \mathrm{w})= \begin{cases}\left(a_{3} / a_{2}\right)^{-4}\left(a / a_{3}\right)^{-2.6}, & a_{3} \leq a<a_{\text {max }} \\
0, z>z_{\beta \max } & \end{cases}
\end{aligned}
$$

And the Novarini- $\gamma$ can be written as:

$$
\begin{aligned}
& \mathrm{N}_{\gamma}=6.0 \times 10^{11} \mathrm{~m}^{-4} \\
& \mathrm{G}_{\gamma}(\mathrm{a}, \mathrm{z})=\left\{\begin{array}{cc}
0, & a<a_{\min } \\
\left(a / a_{1}\right)^{3}, & a_{\min } \leq a<a_{1} \\
1, & a_{1} \leq a<a_{2} \\
\left(a / a_{2}\right)^{-4}, & a_{2} \leq a<a_{3} \\
\left(a_{3} / a_{2}\right)^{-4}\left(a / a_{3}\right)^{-p}, & a_{3} \leq a<a_{\max }
\end{array}\right.
\end{aligned}
$$

where $a_{\min }=10(\mu m), a_{1}=15(\mu m), a_{2}=20(\mu m), a_{3}=54.4+1.984 z(\mu m), a_{\max }=1000(\mu m)$, $\mathrm{z}_{\beta \max }=1.23 \times 10^{-2} \mathrm{w}^{2}, \mathrm{~d}_{\gamma}=0.6(\mathrm{w}-5)+3.5$. The Novarini model with $\beta$-distribution and $\gamma$-distribution was plotted in Fig.1.

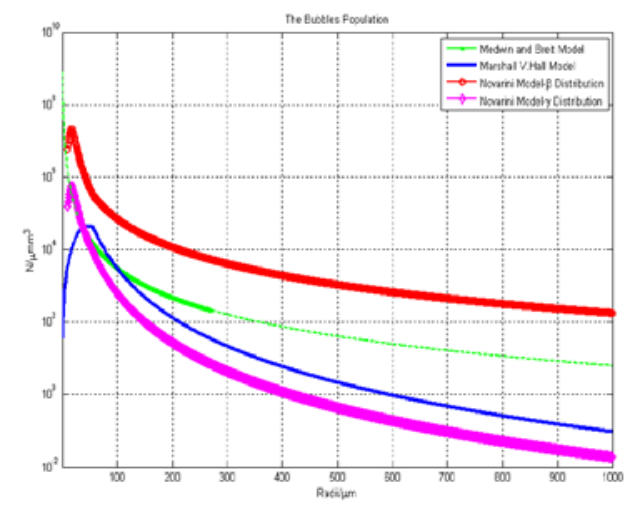

Fig. 1 The bubble-size spectra (depth=1m, w=13m/s)

It is noted that different bubble-size spectra have their own coverage. The four models were plotted in Fig.1, the Medwin-Breit model is extent to $10 \mu \mathrm{m} \sim 1000 \mu \mathrm{m}$. The coverage of Marshall V. Hall model is $16 \mu \mathrm{m} \sim 1000 \mu \mathrm{m}$ and that of Novarini model between $10 \mu \mathrm{m}$ and $1000 \mu \mathrm{m}$. So the estimation must be done carefully with the bubbles distribution. 


\section{The estimation of bubble-size spectra}

\section{Bubble-size spectra using bubble resonance}

If the bubbles were assumed distributing uniformly and independently, the attenuation of sound propagation within bubbly water can be expressed by a function of bubble-size spectra.

$$
\alpha(f)=\frac{c_{0}}{f} \int_{R_{0}=0}^{\infty} \frac{a \delta n(z, a) d a}{\left(f_{0}^{2} / f^{2}-1\right)^{2}+\delta^{2}} \times 20 \lg (\mathrm{e})
$$

where $\delta$ is the bubble damping coefficient and is a function of sound frequency and bubble size and depth, mainly composed of thermal damping, radiated damping and viscous damping[5]. When the sound wave with frequency $f$ illuminate the bubble with radius $a$ at the depth $z$, the damping coefficient could be written as:

$$
\delta=\frac{a_{r}^{2}}{a^{2}} \frac{\operatorname{Im}(B)}{\operatorname{Re}(B)}+\frac{\omega a}{c_{0}}+\delta_{v}
$$

In Eq.14, the first term is the component due to heat diffusion; the second term is the component due to radiation; and the third term is due to the molecular viscosity of seawater. $a_{r}$ is the bubble resonant radius corresponding to the frequency of the sound wave, which can be derived by:

$$
\operatorname{Re}\left[B\left(\phi a_{r}\right)\right]-\rho\left(2 \pi f a_{r}\right)^{2} / 3=0
$$

where $c_{0}$ is the speed of sound in bubble-free water, $B$ is the bulk modulus of elasticity of the bubble, expressed as:

$$
B=\gamma P_{0} /\left(1-3 i(\gamma-1) / 2 \phi^{2} a^{2}[(1+i) \phi a \operatorname{coth}((1+i) \phi a)-1]\right)
$$

In addition to being a function of depth, $B$ is also a function of radius $a$ and frequency $f$. In Eq.16, the $\gamma$ is the ratio of specific heats of the gas in the bubble, $\gamma=1.4, P_{0}$ is the hydrostatic pressure at the bubble, and $\phi=\sqrt{\pi \omega / 2 \pi D}$, in which $D=\mathrm{K}_{\text {gas }} /\left[\rho_{\text {gas }}(R) C_{P}\right]$ is the thermal diffusivity of the gas, $\mathrm{K}_{\text {gas }}$ is the thermal conductivity of the gas and can be simplified as a function of temperature, $\mathrm{K}_{\mathrm{gas}}=A+B T+C T^{2}, A=1.52 \times 10^{-3}, B=7.2342 \times 10^{-5}, C=-9.2207 \times 10^{-9} ; C_{P}$ is the specific heats of the gas at constant pressure, in units of $\mathrm{J} / \mathrm{kg} / \mathrm{K}$, rather than in units of $\mathrm{J} / \mathrm{mol} / \mathrm{K}$, here the $D$ is simplified to a constant $1.84 \times 10^{-5} \mathrm{~m}^{2} / \mathrm{s}$. The last term $\delta_{v}$ in Eq.14 is generally denoted using:

$$
\delta_{v}=4 \mu /\left(\rho \omega a^{2}\right)
$$

which is the coefficient of molecular viscosity of seawater, where $\mu \approx 1.4 \times 10^{-3} \mathrm{~kg} / \mathrm{m} / \mathrm{s}$.

Therefore, the resonant frequency and the resonant radius of bubble using Eq.15 by numerical methods can be obtained. When the depth of bubble and the frequency of sound wave were determined, the damping of bubble can be further derived using Eq.14. A typical of damping coefficient shown in Fig.2, where the sound frequency is $10 \mathrm{kHz}$, and the depth of bubbles is 5 meter beneath surface. It comes out that when the radius of bubble less than $470 \mu \mathrm{m}$, the thermal damping is the main component of the sum damping coefficient, but the radiated damping will be the main component when the radius of bubble larger than $470 \mu \mathrm{m}$. The sum of damping coefficient decreases at first, and then increases very slowly with the radius of the bubble. Thus the damping coefficient is frequently treated as a constant.

Based on Eq.13, the bubble-size spectra estimation was presented by Medwin, only taking the resonant bubbles into consideration and the damping coefficient as a constant and ignoring the surface tension of bubbles. The estimated bubble-size spectra can be expressed:

$$
n_{b}\left(z, R_{0}\right) \approx 4.6 \times 10^{-6} f^{3} \alpha(f) /(1+0.1 \times z)
$$

where $\alpha(f)$ is the attenuation of sound propagation in bubbly water, $\mathrm{z}$ is the depth of bubbles. So, if the attenuation of sound propagation is known, the bubble-size spectra can be determined with Eq.18. 


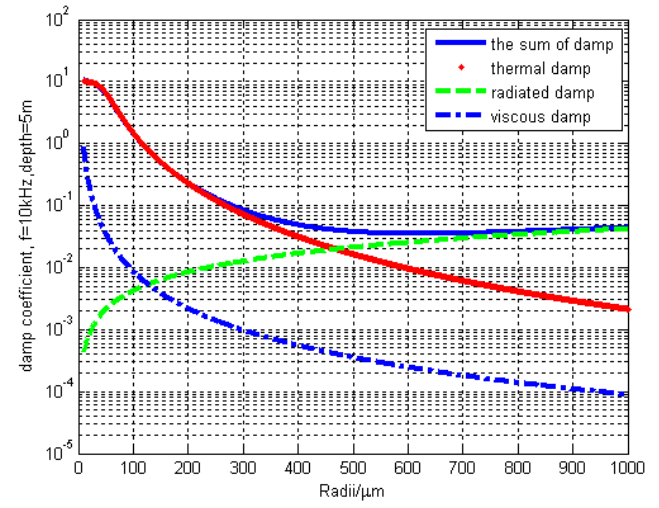

Fig.2 The damp coefficient of bubbles $(\mathrm{f}=10 \mathrm{kHz}, \mathrm{z}=5 \mathrm{~m})$

\section{Bubble-size spectra estimation using Iterative approach}

To reduce the error in the estimation by Eq.18, an iterative approach was adopted for determining the bubble-size spectra. The Eq.13 and Eq.18 were shortly rewritten as:

$$
\begin{aligned}
& \alpha(f)=F T(n(z, a)) \\
& n(z, a)=R B A[\alpha(f)]
\end{aligned}
$$

where the operator $F T$ denotes the process for calculating the sound propagation attenuation in bubbly water with a known bubble-size spectra, and the inverse process is depicted by the operator $R B A$.The sound propagation attenuation at interesting frequency with a known bubble-size spectra $n_{t}$ can be expressed by:

$$
\alpha_{t}(f)=F T\left(n_{t}\right)
$$

While, the estimated bubble-size spectra can be denoted by:

$$
n_{0}=R B A\left[\alpha_{t}(f)\right]
$$

So, the estimation error is written as

$$
n_{\varepsilon}=n_{t}-n_{0}
$$

In order to obtain more accurate bubble-size spectra estimation, the error $n_{\varepsilon}$ should be further investigated, and the two operators FT and RBA were done on the estimation bubble-size spectra $n_{0}$ again.

So

$$
\begin{aligned}
& \alpha_{0}(f)=F T\left(n_{0}\right) \\
& n_{1}=R B A\left[\alpha_{0}(f)\right]
\end{aligned}
$$

$$
n_{\varepsilon}^{\prime}=n_{0}-n_{1}=R B A\left[F T\left(n_{\varepsilon}\right)\right]
$$

Since the estimation error $n_{\varepsilon}$ is the first-order small term, the estimation error $n_{\varepsilon}^{\prime}$ is the second-order small term, and the two operators FT and RBA represent forward and inverse process, Eq.26 becomes:

$$
\operatorname{RBA}\left[F T\left(n_{\varepsilon}\right)\right]=n_{t}-n_{0}
$$

Above all, it can be obtained that the second-order approximation of bubble-size spectra can be expressed as:

$$
n_{t}=2 n_{0}-n_{1}
$$

Of course, if the second-order approximation could not still meet requirements, the higher order approximation can be similarly done.

\section{The estimation error analysis of bubble-size spectra}

Although two methods for estimating bubble-size spectra have been developed, the issue of estimation accuracy remains a problem to investigate. Unfortunately, we do not have any known 
bubble-size spectra and propagation attenuation samples to exam the two methods, so that a compromise was made on the issue of estimation accuracy by a known bubble-size spectra, and the approximated sound propagation attenuation were gained using Eq.13. Based on this notion, the accuracy of bubble-size spectra could be investigated.

The four bubble-size spectra models above were adopted, and then obtain the approximated sound propagation attenuation using Eq.13, where the integration is achieved by numerical solution with $1 \mu \mathrm{m}$ interval. The four bubble-size spectra were estimated using Eq.18 and Eq.27 respectively, plotted in Fig.3. The dashed lines denoted the known bubble-size spectra, and the dotted lines represented the estimations of the known bubble-size spectra with bubble resonance, the solid lines indicated the estimations of the known bubble-size spectra with the iterative approach. It was shown that the main errors happened at the two-end of the bubble-size spectra and the performance of bubble-size spectra estimation with the iterative approach was wholly better than that with bubble resonance, and the estimation errors were plotted in Fig.4, The dashed lines denoted the bubble-size spectra estimation errors with bubble resonance, the solid lines represented bubble-size spectra estimation errors with the iterative approach. The conclusion obtained above were verified apparently and more detail information shown in Table I.

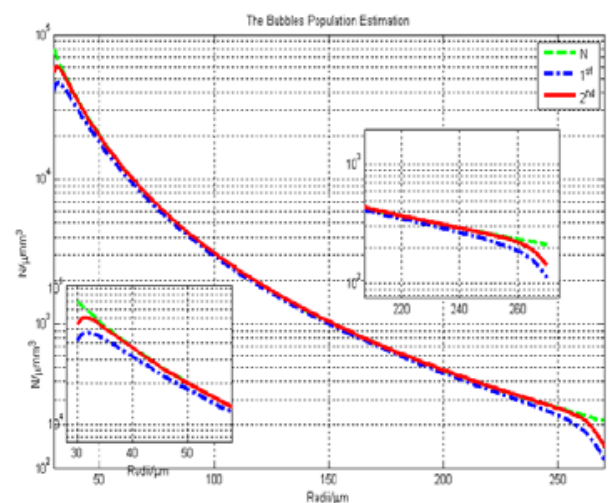

(a)

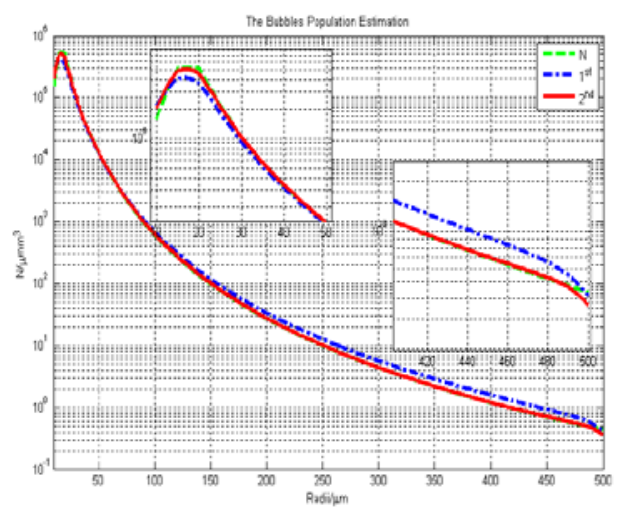

(c)

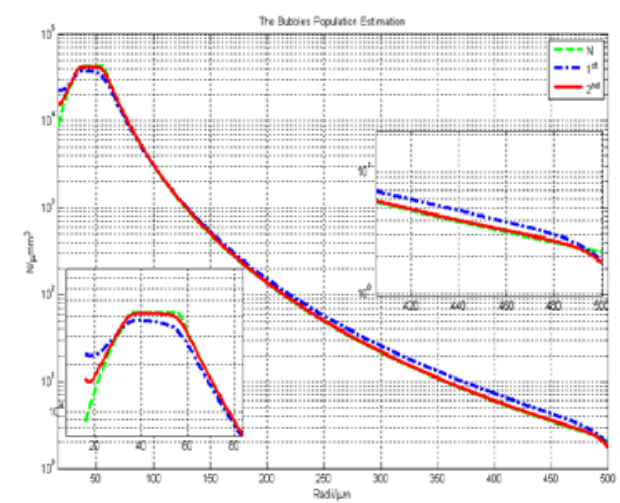

(b)

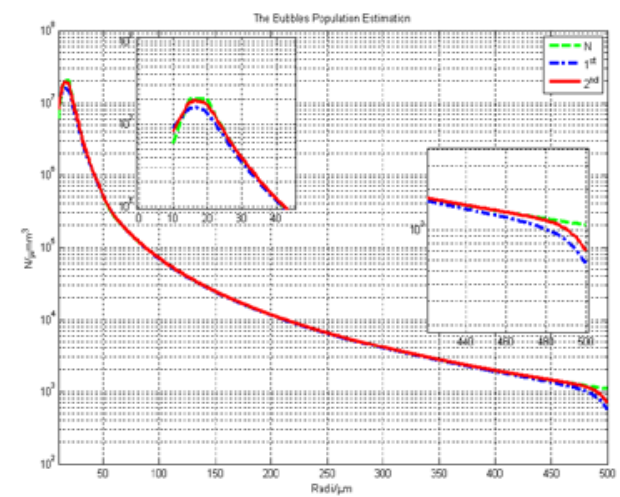

(d)

Fig.3 The bubble-size spectra estimation with Medwin-Breit (a), Marshall V. Hall(b), Novarini- $\gamma$ Model(c), Novarini- $\beta(d)$ Model 


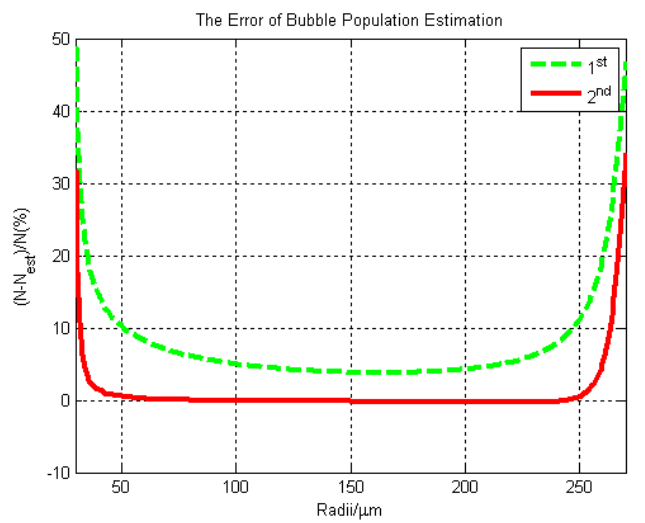

(a)

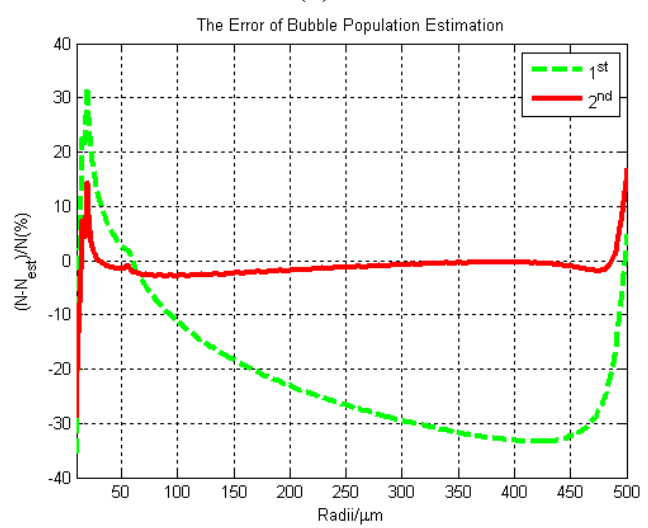

(c)

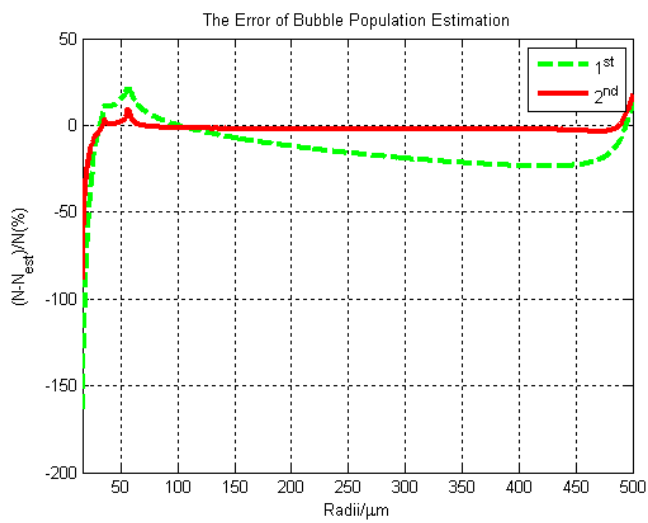

(b)

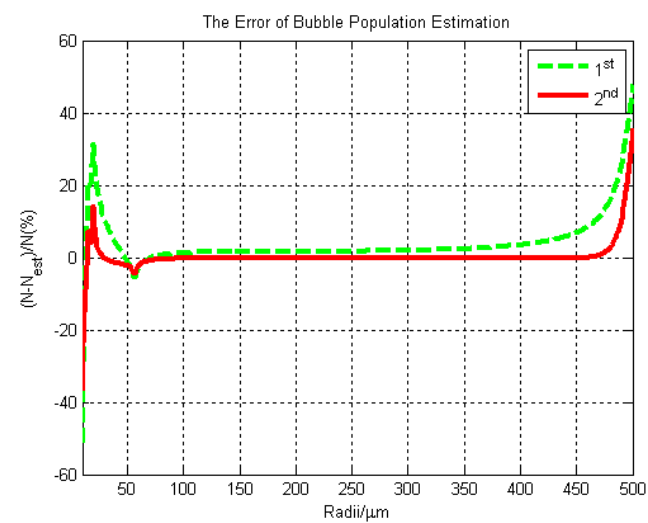

(d)

Fig.4 The estimation error of bubble-size spectra with Medwin-Breit (a), Marshall V. Hall(b), Novarini- $\gamma$ Model(c), Novarini- $\beta(d)$ Model

Table I The results of bubble-size spectra estimation error

\begin{tabular}{|c|c|c|c|c|c|c|c|}
\hline \multirow{2}{*}{$\begin{array}{l}\text { Bubble-size } \\
\text { spectra }\end{array}$} & \multicolumn{2}{|c|}{ The mean of error } & \multicolumn{2}{|c|}{$\begin{array}{c}\text { The standard deviation of } \\
\text { error }\end{array}$} & \multicolumn{2}{|c|}{ The max error and its radius } & \multirow{2}{*}{ Coverage } \\
\hline & $\begin{array}{l}\text { bubble } \\
\text { resonance }\end{array}$ & $\begin{array}{l}\text { iterative } \\
\text { approach }\end{array}$ & $\begin{array}{l}\text { bubble } \\
\text { resonance }\end{array}$ & $\begin{array}{l}\text { iterative } \\
\text { approach }\end{array}$ & $\begin{array}{l}\text { Bubble } \\
\text { resonance }\end{array}$ & $\begin{array}{l}\text { iterative } \\
\text { approach }\end{array}$ & \\
\hline $\begin{array}{l}\text { Medwin-Breit } \\
\text { model }\end{array}$ & $8.00 \%$ & $1.13 \%$ & $7.46 \%$ & $4.69 \%$ & 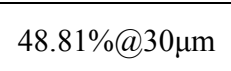 & $34.14 \% @ 270 \mu \mathrm{m}$ & $30 \mu \mathrm{m} \sim 270 \mu \mathrm{m}$ \\
\hline $\begin{array}{l}\text { Marshall V. } \\
\text { Hall model }\end{array}$ & $13.26 \%$ & $2.12 \%$ & $15.57 \%$ & $5.91 \%$ & 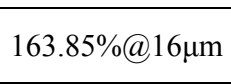 & 89.18\%@16um & $16 \mu \mathrm{m} \sim 500 \mu \mathrm{m}$ \\
\hline $\begin{array}{l}\text { Novarini- } \gamma \\
\text { model }\end{array}$ & $20.95 \%$ & $1.11 \%$ & $13.36 \%$ & $2.72 \%$ & 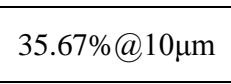 & 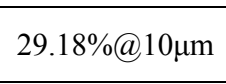 & $10 \mu \mathrm{m} \sim 500 \mu \mathrm{m}$ \\
\hline $\begin{array}{l}\text { Novarini- } \beta \\
\text { model }\end{array}$ & $4.03 \%$ & $0.32 \%$ & $7.46 \%$ & $4.30 \%$ & 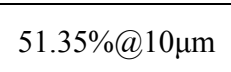 & 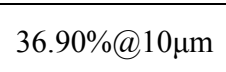 & $10 \mu \mathrm{m} \sim 500 \mu \mathrm{m}$ \\
\hline
\end{tabular}

The comparison in Table I show that the estimation error means of bubble-size spectra with the iterative approach are less than $3 \%$, while as much as $20 \%$ with the bubble resonance, meanwhile the iterative approach has smaller variance than the bubble resonance. Although the performance of bubble-size spectra estimation at the two-end is improved limitedly, the performance of bubble-size spectra estimation with iterative approach is wholly better than that with bubble resonance.

\section{Summary}

The four bubble-size spectra models and the estimation of bubble-size spectra were described briefly in this paper, which was used to research the accuracy of the bubble-size spectra estimation. It comes out that the bubbles with medium radii can be better determined than the bubbles distributed at the two-end and the iterative approach is wholly better for estimating bubble-size spectra than the bubble resonance and with less variation of the estimation error. Therefore the iterative approach has a better performance for determining bubble-size spectra than the resonance. 


\section{References}

[1] Eric Lamarre, W.K. Melville, Sound-speed measurements near the ocean surface, Journal of the Acoustical of American, Vol. 96(1994), p.3605

[2] Bok Kyoung Choi and Suk Wang Yoon, Acoustic bubble counting technique using sound speed extracted from sound attenuation, Jourmal of Oceanic Engineering, Vol.26(2001),p.125

[3] Valentin Leroy, Anatoliy Strybulevych,John H.Page and Martin G.Scanlon,Sound velocity and attenuation in bubbly gels measured by transmission experiments, Journal of the Acoustical of American, Vol. 123(2008), p.1931

[4] Charles Devin, Survey of thermal, radiation, and viscous damping of pulsating air bubbles in water, Journal of the Acoustical of American,Vol.31(1959),p.1654

[5] Marshall V. Hall, A comprehensive model of wind-generated bubbles in the ocean and predictions of the effects on sound propagation at frequencies up to $40 \mathrm{kHz}$, Journal of the Acoustical of American,Vol.86(1989),p.1103

[6] Michael A. Ainsile and Timothy G. Leighton, Review of scattering and extinction cross-section, damping factors, and resonance frequencies of a spherical gas bubble, Journal of the Acoustical of American,Vol.130(2011),p.3184

[7] Herman Medwin, Acoustic determinations of bubble-size spectra, Journal of the Acoustical of American,Vol.62(1977) ,p.1041

[8] J. W. Caruthers, P. A. Elmore, An iterative approach for approximating bubble distributions form attenuation mearsurements, Journal of the Acoustical of American,Vol.106(1999),p. 185

[9] Bok Kyoung Choi and Suk Wang Yoon, Acoustic bubble counting technique using sound speed extracted from sound attenuation, Journal of Oceanic Engineering,Vol. 26(2001),p.125

[10] Thomas C. Weber, Anthony P. Lyons, and David L. Bradley, Acoustic Propagation Through Clustered Bubble Clouds, Journal of Oceanic Engineering,Vol. 32(2007),p.513

[11] Jorge C. Novarini, Richard S. Keiffer, and Guy V. Norton, A model for variations in the range and depth dependence of the sound speed and attenuation induced by bubble clouds under wind-driven sea surface, Jourmal of Oceanic Engineering,Vol. 23(1998),p. 423

[12] B.R.Rapids and R.L. Culver, An acoustic ship wake for propagation studies, Applied Research Laboratory Technical Memorandum, File No. 00-068, 2000.

[13] Mark V. Trevorrow, Svein Vagle and David M. Farmer, Acoustic measurements of microbubbles within ship wakes, Journal of the Acoustical of American,Vol.95(1994),p.1922 